\title{
新型苯并噻唑离子液体的合成及其催化蓖麻油酸酯化研究
}

\author{
张海飞刘冬梅康婷婷王也张小祥* 朱新宝* \\ (南京林业大学化学工程学院 南京 210037)
}

\begin{abstract}
摘要 以价格低廉的苯并噻唑为原料, 合成了 4 种新型功能化布朗斯特酸性离子液体: 苯并噻唑硫酸氢盐([HBth]$\left.\mathrm{HSO}_{4}\right)$ 、苯并噻唑磷酸二氢盐 $\left([\mathrm{HBth}] \mathrm{H}_{2} \mathrm{PO}_{4}\right)$ 、苯并噻唑高氯酸盐 $\left([\mathrm{HBth}] \mathrm{ClO}_{4}\right)$ 、苯并噻唑硝酸盐 $\left([\mathrm{HBth}] \mathrm{NO}_{3}\right)$, 其结构经 FT-IR、HRMS、NMR 表征得到确认. 考察了 4 种酸性离子液体在菎麻油酸和乙二醇丁醚(EGBE)酯化反应中的催化活 性, 并与以浓硫酸为催化剂的催化体系相对照. 结果表明: 当 $n$ (菎麻油酸) $: n$ (乙二醇丁醚) $=1: 1.15$, 离子液体催化剂 用量为菎麻油酸重量的 $2 \%$, 反应温度 $90{ }^{\circ} \mathrm{C}$, 反应时间 $5 \mathrm{~h}$, 酯化率可达 $98.8 \%$, 离子液体催化剂经真空干燥重复使用 10 次后, 仍具有较高的催化活性. 还考察了以苯并噻唑硫酸氢盐为催化剂催化合成系列葲麻油酸二元醇醚酯和短链脂 肪酸酯, 也获得了较高的酯化率, 且离子液体均能与酯产物自动分相. 与传统硫酸催化酯化相比, 此类离子液体催化 酯化具有生产成本低、过程清洁、使用周期长等优点, 完全可以替代传统浓硫酸催化葛麻油酸酯化反应.
\end{abstract}

关键词 苯并噻唑; 离子液体; 菎麻油酸; 酯化反应; 催化活性

\section{Synthesis of Novel Benzothiazolium Ionic Liquids and Research on Their Catalytic Esterification for Ricinoleic Acid}

\author{
Zhang, Haifei \\ Liu, Dongmei \\ Kang, Tingting \\ Wang, Ye \\ Zhang, Xiaoxiang* \\ Zhu, Xinbao* \\ (College of Chemical Engineering, Nanjing Forestry University, Nanjing 210037)
}

\begin{abstract}
Four new task-specfic benzothiazolium ionic liquids (ILs) [HBth] $\mathrm{HSO}_{4},[\mathrm{HBth}] \mathrm{H}_{2} \mathrm{PO}_{4},\left[\mathrm{HBth} \mathrm{ClO}_{4},\left[\mathrm{HBth} \mathrm{NO}_{3}\right.\right.$ were synthesized and characterized by NMR and FT-IR, which bear the proton acid groups based on low price benzothiazole as raw material. The catalytic activities of these acidic ionic liquids were investigated by the esterification of ricinoleic acid with ethanol ethylene glycol monobutyl ether (EGBE) and were compared with concentrated sulfuric acid. The results showed that under the optimized conditions: $n$ (Ricinoleic acid) $: n(\mathrm{EGBE})=1: 1.15$, catalyst dosage $2 \%$ (wt) of ricinoleic acid, reaction temperature $90{ }^{\circ} \mathrm{C}$ and reaction time $5 \mathrm{~h}$, esterification yield was up to $98.8 \%$. The ionic liquids dried in vacuum till remained high activity after reused for 10 times. Finally, the yields of series of ricinoleic acid binary alcohol ether ester and short-chain fatty acids ester were also high with $[\mathrm{HBth}] \mathrm{HSO}_{4}$ as catalyst, and the ionic liquids can form split-phases with ester product. Compared with the traditional sulfuric acid catalytic esterification, catalytic esterification of such ionic liquid has the advantages of low production cost, clean process, low corrosion rate, reusable, and has the potential of replacing the traditional sulfuric acid in catalytic esterification reaction of ricinoleic acid.
\end{abstract}

Keywords benzothiazolium; ionic liquids; ricinoleic acid; esterification; catalytic activity

蓖麻油分子中含有双键、羟基、酯基等活性官能团, 可发生水解、加成、酯交换等诸多化学反应，已被成功 开发成数百种精细化工基本原料 ${ }^{[1 \sim 4]}$. 以菎麻油为原料 合成的菎麻油酸甲酯、葛麻油酸正丁酯以及㵧麻油酸甘 油酯是一系列非常重要的、优质的增塑剂，具有可生物 降解、耐低温等特性, 有着广阔的应用前景 ${ }^{[5,6]}$.
蓖麻油酸酯化的传统工艺是采用浓硫酸催化酯化, 该法引起的副反应多、产物收集过程复杂，其工业化过 程中使用的浓硫酸催化剂对设备严重腐蚀，易造成三废 污染，因此寻找绿色清洁的催化剂来代替浓硫酸尤为重 要 ${ }^{[7 ~ 11]}$.

近年来，离子液体作为一种温和、绿色反应介质，

\footnotetext{
*E-mail:s070038@hotmail.com; zhuxinbao@njfu.com.cn

Received November 12, 2015; revised December 21, 2015; published online January 15, 2016.

Project supported by the 948 Project Foundation of State Forestry Administration (No. 2015-4-55).

国家林业局 948 项目基金(No. 2015-4-55)资助项目.
} 
在物质分离、电化学、催化剂、有机合成、材料制备等 领域得到了广泛的应用 ${ }^{[12 ~ 15]}$. 离子液体作为一种新型 的环境友好型溶剂和液体酸催化剂, 它同时拥有液体酸 的高反应活性和固体酸的不挥发性的优点 ${ }^{[16 ~ 18]}$, 而且 其分子结构和酸性具有可调控性, 催化剂和产物易分 离, 热稳定性高. 由于离子液体所具有的独特性能, 咪 唑基 ${ }^{[19]}$ 、吡啶基等传统离子液体被广泛应用于化学研究 的多个领域中, 特别是用于各类酯化反应中 ${ }^{[20,21]}$ 得到了 较好的催化效果, 但是这些新型催化剂大多数伴随着一

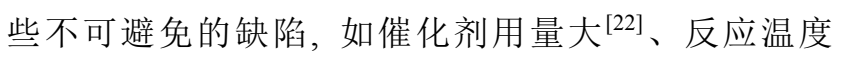
高 ${ }^{[23]}$ 、催化效果不佳、价格昂贵、有毒等. 寻找高效、 廉价的催化剂是当前离子液体催化剂研究领域的又一 热点 ${ }^{[24,25]}$. 苯并噻唑具有与咪唑、吡啶的类似结构, 是 潜在的离子液体的原料, 其价格远低于咪唑和吡啶, 根 据离子液体的可设计性, 可以开发出符合要求的新型苯 并噻唑基离子液体.

本文以价格低、毒性小的苯并噻唑为原料与四种无 机酸经反应合成了 4 种新型的苯并噻唑酸性离子液体 (Eq. 1, 图 1), 经仔细查阅文献, 此类化合物未见报道. 考察了其催化合成葛麻油酸乙二醇丁醚酯的催化活性, 并对酯化反应条件进行了优化. 重点考察了苯并噻唑硫 酸氢盐这一离子液体对系列葛麻油酸二元醇梄酯的催 化合成活性研究, 结果表明其催化效果非常突出. 该离 子液体克服了传统催化剂存在的缺点 ${ }^{[26]}$, 提高了催化 效率, 降低了制备成本, 有望代替传统酸性催化剂, 具 有广阔的应用前景 ${ }^{[27 ~ 31] . ~}$

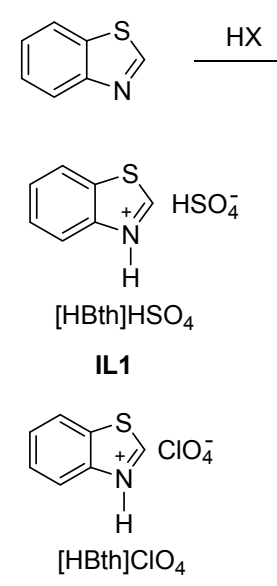

IL3

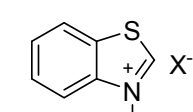

!

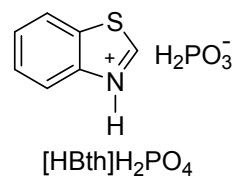

IL2

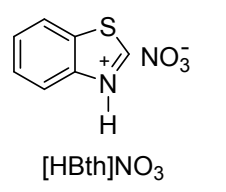

IL4
图 1 离子液体结构示意图

Figure 1 Structures of ionic liquids

\section{1 结果与讨论}

\section{1 蓖麻油酸二元醇醚酯的合成以及酯化率的计算}

在装有搅拌器、分水器、回流冷凝管和温度计的三
口烧瓶中依次加入 $29.8 \mathrm{~g}(0.1 \mathrm{~mol})$ 的蓖麻油酸、 $13.5 \mathrm{~g}$ $(0.115 \mathrm{~mol})$ 乙二醇丁醚、 $0.6 \mathrm{~g}$ (菎麻油酸重量的 $2 \%$ ) 苯并 噻唑离子液体催化剂和环已烷 $50 \mathrm{~mL}$, 加热回流带水反 应 $5 \mathrm{~h}$, 直至水不再生成后冷却, 采用国标 GB1668-81 测定反应混合液的酸值, 酯化率的计算公式如下所示:

酯化率 $=(1$-反应后溶液中酸的质量/反应前溶液 中酸的质量) $\times 100 \%$

反应结束后, 先冷却过滤出催化剂, 再用饱和碳酸 钠溶液中和, 分出有机层, 用无水硫酸镁干燥, 过滤后 将粗产物进行减压蒸馏, 得到蓄麻油酸乙二醇丁梄酯 (Eq. 1).

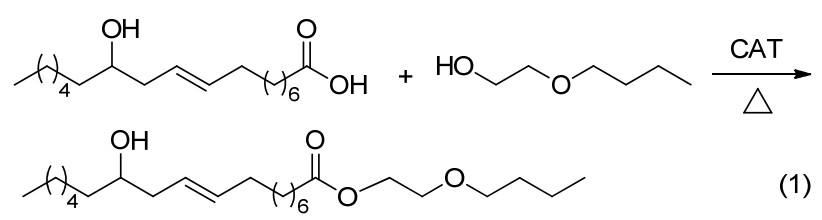

用类似方法合成其它系列蓖麻油酸二元醇醚酯和 短链脂肪酸酯.

\section{2 离子液体的酸性分析}

酸性离子液体不仅具有液体酸的高反应活性位，而 且具有固体酸的不挥发性. 酯化反应作为酸催化的可逆 反应,

其反应进行的程度与催化剂的酸性强弱有直接的 关系, 为了探索所合成的离子液体催化剂的酸性与催化 活性的对应关系, 测试了不同浓度(质量分数)的离子液 体以及浓硫酸水溶液的 $\mathrm{pH}$ 值, 结果如表 1 .

表 1 不同离子液体水溶液酸性 ${ }^{a}$

Table 1 The acidity of various ionic liquids in water

\begin{tabular}{lccccc}
\hline \multirow{1}{*}{\multicolumn{1}{c}{ IL }} & \multicolumn{5}{c}{$\mathrm{pH}$} \\
\cline { 2 - 6 } & $1 \%$ & $2 \%$ & $3 \%$ & $4 \%$ & $5 \%$ \\
\hline$[\mathrm{HBth}] \mathrm{HSO}_{4}$ & 1.33 & 1.28 & 1.05 & 0.80 & 0.68 \\
{$[\mathrm{HBth}] \mathrm{H}_{2} \mathrm{PO}_{4}$} & 1.58 & 1.43 & 1.37 & 1.11 & 0.89 \\
{$[\mathrm{HBth}] \mathrm{ClO}_{4}$} & 1.38 & 1.31 & 1.24 & 0.89 & 0.70 \\
{$[\mathrm{HBth}] \mathrm{NO}_{3}$} & 1.41 & 1.35 & 1.27 & 0.96 & 0.83 \\
$\mathrm{H}_{2} \mathrm{SO}_{4}$ & 1.32 & 1.26 & 1.02 & 0.80 & 0.65 \\
\hline
\end{tabular}

由表 1 可知, 对同一种溶液, 其 $\mathrm{pH}$ 值随着质量浓度 的增大而降低, 因为水有高的介电常数以及很强的与离 子液体阴离子形成氢键的能力, 从而有效地解离离子液 体的聚集体; 离子液体 $[\mathrm{HBth}] \mathrm{HSO}_{4}$ 和浓硫酸的水溶液 的 $\mathrm{pH}$ 值相近, 说明该离子液体酸性强弱与氮杂环结构 没有呈现明显的相关性; 四个阳离子部分相同的离子液 体, 其 $\mathrm{pH}$ 值总体上随阴离子的共轭酸酸性的增强而减 小, 但 $\left[\mathrm{HBth}^{-} \mathrm{ClO}_{4}\right.$ 的 $\mathrm{pH}$ 值大于 $[\mathrm{HBth}] \mathrm{HSO}_{4}$, 这是由于 $[\mathrm{HBth}] \mathrm{HSO}_{4}$ 阴离子部分 $\mathrm{HSO}_{4}^{-}$电离产生 $\mathrm{H}^{+}$, 增强了酸 性, 使得 $\mathrm{pH}$ 值减小, 说明离子液体的酸性受到阴阳离 
子两部分的共同影响. 通过对离子液体酸强度的测试, 为后续对催化剂催化性能的研究提供了理论依据.

\section{2 稳定性测试}

利用 STD-2960 型差热-热重联用热分析仪测试离 子液体的热稳定性, 在氮气保护及升温速率为 $10{ }^{\circ} \mathrm{C} /$ $\min$ 的条件下测定了 4 种离子液体的热稳定性, 结果见 图 2.

由图 2 可见, $100 \sim 230{ }^{\circ} \mathrm{C}$ 有少量失重, 这是由于样 品失水造成的, 离子液体在分解前, 热重曲线较平坦, 4 种苯并噻唑离子液体的分解温度都大于 $250{ }^{\circ} \mathrm{C}$, 说明 制备的离子液体比较稳定. 离子液体的分解温度主要取 决于其阴、阳离子结构之间作用力及其本身含水量, 作 用力越大, 含水量越小, 离子液体越稳定. 在失重过程 中没有原料及其它杂质峰出现, 所制备的离子液体几乎 不挥发, 说明所制备的离子液体较纯. 酯化反应一般在 低于 $250{ }^{\circ} \mathrm{C}$ 条件下进行, 故合成的这 4 种新型催化剂能 够在酯化反应体系中稳定存在, 并发挥催化作用, 为醇 酸酯化反应提供一个较大的操作温度范围.
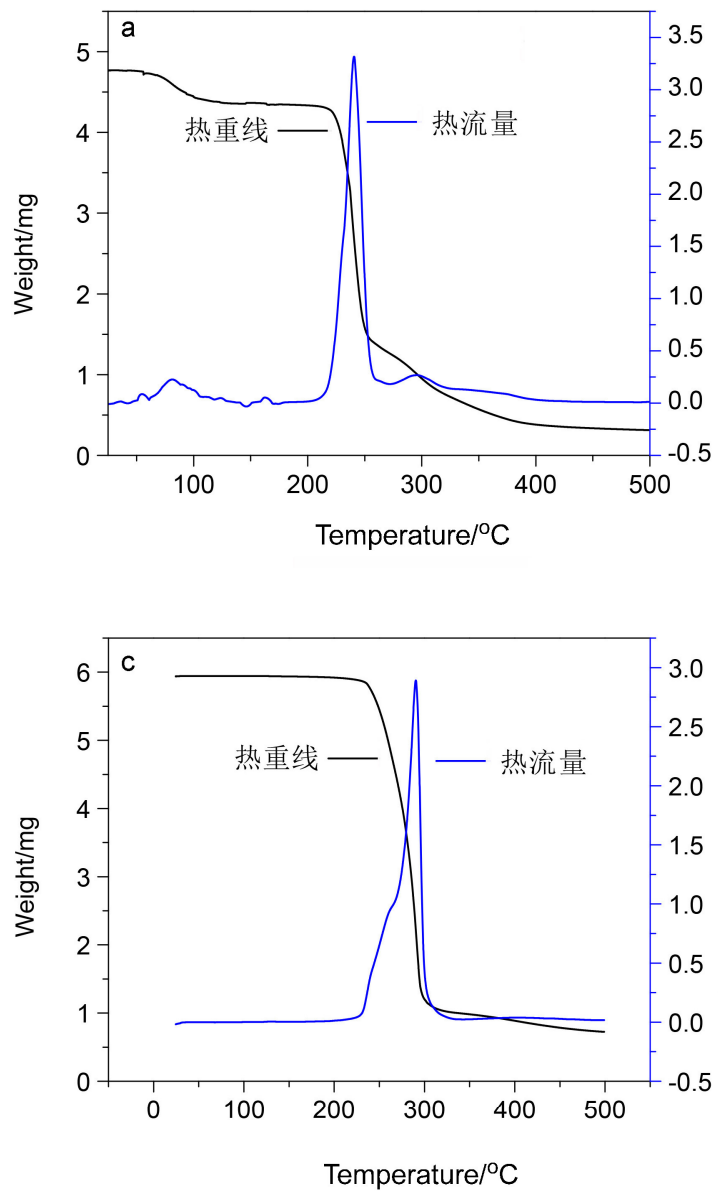

\section{3 溶解性测定}

分别以水、甲醇、乙醇、正丙醇、氯仿、乙醚、正 己烷、乙酸乙酯以及甲苯为溶剂，考察离子液体的溶解 性情况，溶解度标准根据《中国药典》2010 版采用易溶、 溶解、略溶、微溶、几乎不溶或不溶来描述药品在不同 溶剂中溶解性能，结果见表 2 .

表 2 离子液体的溶解性 ${ }^{a}$

Table 2 Solubility of the ionic liquids ${ }^{a}$

\begin{tabular}{lccccc}
\hline \multicolumn{1}{c}{ 溶剂 } & IL1 & IL2 & IL3 & IL4 & $\mathrm{H}_{2} \mathrm{SO}_{4}$ \\
\hline Water & f.s & f.s & f.s & f.s & f.s \\
Methanol & f.s & f.s & f.s & f.s & f.s \\
Ethanol & f.s & f.s & f.s & f.s & f.s \\
Isopropanol & $\mathrm{s}$ & $\mathrm{s}$ & $\mathrm{s}$ & $\mathrm{s}$ & $\mathrm{s}$ \\
Trichloromethane & $\mathrm{s}$ & $\mathrm{s}$ & $\mathrm{s}$ & $\mathrm{s}$ & $\mathrm{i}$ \\
Ether & $\mathrm{i}$ & $\mathrm{i}$ & $\mathrm{i}$ & $\mathrm{i}$ & $\mathrm{s}$ \\
Cyclohexane & $\mathrm{i}$ & $\mathrm{i}$ & $\mathrm{i}$ & $\mathrm{i}$ & $\mathrm{i}$ \\
Ethyl acetate & $\mathrm{i}$ & $\mathrm{i}$ & $\mathrm{i}$ & $\mathrm{i}$ & $\mathrm{s}$ \\
Methanylbenzene & $\mathrm{i}$ & $\mathrm{i}$ & $\mathrm{i}$ & $\mathrm{i}$ & $\mathrm{i}$ \\
\multicolumn{2}{c}{ Test conditions: $V_{\text {solven }}=5 \mathrm{~mL}, m\left(\mathrm{IL}\right.$ or $\left.\mathrm{H}_{2} \mathrm{SO}_{4}\right)=0.2 \mathrm{~g}$, f.s: freely soluble, s: } \\
soluble, s.s: slightly soluble, i: insoluble.
\end{tabular}
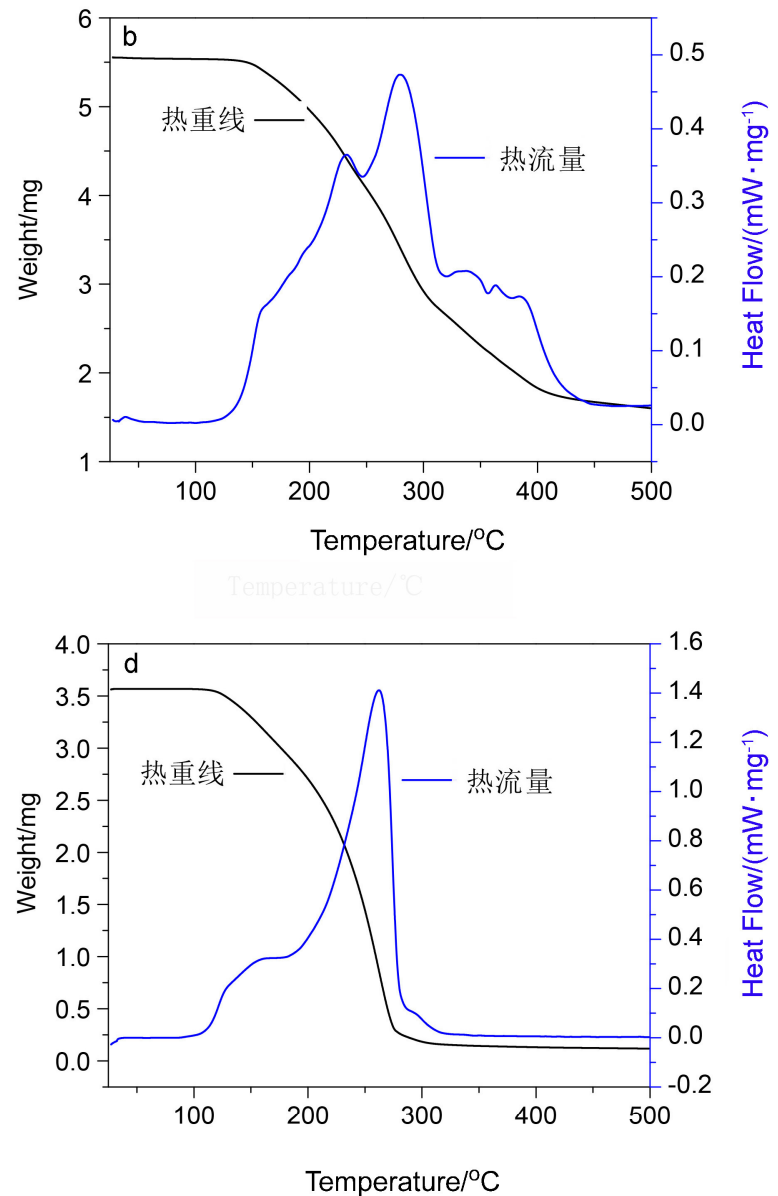

图 2 离子液体(a) $[\mathrm{HBth}] \mathrm{HSO}_{4}$ 、 (b) $[\mathrm{HBth}] \mathrm{H}_{2} \mathrm{PO}_{4}$ 、 (c) $[\mathrm{HBth}] \mathrm{ClO}_{4}$ 和(d) $[\mathrm{HBth}] \mathrm{NO}_{3}$ 的 TG-DSC 图 Figure 2 TG-DSC of ionic liquids $[\mathrm{HBth}] \mathrm{HSO}_{4}(\mathrm{a}),[\mathrm{HBth}] \mathrm{H}_{2} \mathrm{PO}_{4}(\mathrm{~b}),[\mathrm{HBth}] \mathrm{ClO}_{4}(\mathrm{c})$ and $[\mathrm{HBth}] \mathrm{NO}_{3}(\mathrm{~d})$ 
结果表明, 离子液体与非极性溶剂不相溶, 与较强 的极性溶剂互溶. 离子液体溶解范围广, 溶解度大, 能 力强, 具有较强的离子环境, 在许多有机和无机物质中 表现出良好的溶解性, 能够发挥其介质和催化双重功 能. 离子液体的溶解度范围和大小是其本身的结构特征 所决定的. 与浓硫酸不同, 此类离子液体均不溶于有机 羧酸酯, 反应结束后, 它们与产物酯自动分层, 过滤就 可以回收催化剂, 避免碱洗除去催化剂的过程, 不但简 化了后处理催化剂的操作, 而且减少了对环境造成的污 染. 离子液体的溶解性能的研究为离子液体在催化酯化 反应中的应用提供了重要的理论依据.

\section{4 催化剂的催化活性}

为了比较不同催化剂对酯化反应的催化活性, 本文 设计了一系列实验. 实验选择菎麻油酸与乙二醇丁醚为 反应原料, 在反应温度 $90{ }^{\circ} \mathrm{C}, n$ (蓖麻油酸) $: n$ (乙二醇 丁醚 $)=1: 1.15$, 催化剂用量为菎麻油酸总质量的 2 $\mathrm{wt} \%$, 反应时间 $6 \mathrm{~h}$ 的反应条件下, 考察不同酸性离子 液体(ILs)对反应酯化率的影响, 实验结果见表 3 .

表 3 离子液体的催化活性 ${ }^{a}$

Table 3 Effect of different catalysts on results of esterification ${ }^{a}$

\begin{tabular}{clc}
\hline Entry & \multicolumn{1}{c}{ ILs } & Yield $^{b} \%$ \\
\hline 1 & {$\left[\right.$ HBth] $\mathrm{HSO}_{4}$} & 99 \\
2 & {$[\mathrm{HBth}] \mathrm{H}_{2} \mathrm{PO}_{4}$} & 97 \\
3 & {$[$ HBth]ClO } & 98 \\
4 & {$\left[\mathrm{HBth}_{4}\right.$} & 98 \\
5 & $\mathrm{H}_{2} \mathrm{SO}_{4}$ & 98 \\
\hline
\end{tabular}

${ }^{a}$ Reaction conditions: $n$ (ricinoleic acid) $: n(\mathrm{EGBE})=1: 1.15 ; w(\mathrm{IL}$ or $\left.\mathrm{H}_{2} \mathrm{SO}_{4}\right)=2 \%$ of ricinoleic acid; Temperature $=90{ }^{\circ} \mathrm{C}$; Time $=5 \mathrm{~h} ;{ }^{b}$ Esterification yield.

菎麻油酸与乙二醇丁醚反应是典型的酸催化酯化 反应, 催化剂的酸性对反应有很大影响, 不同离子液体 催化剂的酸性强弱各不相同, 其相应的催化能力也有差 别. 在相同的反应条件下, 考察所合成出的 4 种苯并噻 唑酸性离子液体对此反应的催化性能, 并与传统的无机 酸硫酸进行对比, 结果表明: [HBth] $\mathrm{HSO}_{4}$ 离子液体对酯 化反应具有可与浓硫酸相比拟的催化活性, 所合成的 4 个离子液体中, $[\mathrm{HBth}] \mathrm{HSO}_{4}$ 离子液体的催化活性较高, 这与其阴离子的酸性强这一特性相符合. 合成的几种离 子液体均能与酯自动分层, 不仅有利于平衡向反应正方 向进行，促进酯化反应发生，提高反应的酯化率; 而且 还简化了分离过程, 省去用碱中和处理而产生大量废水 的操作步骤, 减少了对环境造成的污染. 此外, 传统催 化剂浓硫酸会使反应产物色泽较深, 氧化等副产物明显 增多, 而用离子液体作催化, 所得的反应产物颜色明显 较浅.

\section{5 正交实验考察 $[\mathrm{HBth}] \mathrm{HSO}_{4}$ 对酯收率的影响}

本实验选用三水平、四因素进行正交实验，四因素 分别为反应温度、反应时间、催化剂用量、原料物质的 量比, 每个因素选择 3 个水平. 评价指标为蓖麻油酸的 酯化率, 选用催化剂为 $[\mathrm{HBth}] \mathrm{HSO}_{4}$, 所设计的正交实验 因素-水平表(表 4)及正交实验与结果(表 5).

表 4 正交实验因素-水平表

Table 4 Levels and factors for orthogonal experiment

\begin{tabular}{ccccc}
\hline Level & Temp. ${ }^{a}{ }^{\circ} \mathrm{C}$ & Time $/ \mathrm{h}$ & $\begin{array}{c}w \text { (ricinoleic } \\
\text { acid) } / \%\end{array}$ & $\begin{array}{c}n(\mathrm{EGBE}): \\
n(\text { ricinoleic acid) }\end{array}$ \\
\hline 1 & 80 & 4 & 1 & $1.10: 1$ \\
2 & 85 & 5 & 2 & $1.15: 1$ \\
3 & 90 & 6 & 3 & $1.20: 1$ \\
\hline
\end{tabular}

表 5 正交实验与结果

Table 5 Results of orthogonal experiment

\begin{tabular}{|c|c|c|c|c|c|}
\hline Factor & Temp. $/{ }^{\circ} \mathrm{C}$ & Time/h & $\begin{array}{l}w(\text { ricinoleic } \\
\text { acid }) / \%\end{array}$ & $\begin{array}{c}n(\text { EGBE }): \\
n(\text { ricinoleic acid })\end{array}$ & Yield $^{b} / \%$ \\
\hline 1 & 80 & 4 & 1 & $1.10: 1$ & 85 \\
\hline 2 & 80 & 5 & 2 & $1.15: 1$ & 92 \\
\hline 3 & 80 & 6 & 3 & $1.20: 1$ & 84 \\
\hline 4 & 85 & 4 & 2 & $1.20: 1$ & 92 \\
\hline 5 & 85 & 5 & 3 & $1.10: 1$ & 98 \\
\hline 6 & 85 & 6 & 1 & $1.15: 1$ & 95 \\
\hline 7 & 90 & 4 & 3 & $1.15: 1$ & 98 \\
\hline 8 & 90 & 5 & 2 & $1.20: 1$ & 98 \\
\hline 9 & 90 & 6 & 1 & $1.10: 1$ & 96 \\
\hline $\mathrm{K} 1$ & 86.65 & 91.42 & 91.74 & 92.60 & - \\
\hline $\mathrm{K} 2$ & 94.80 & 95.80 & 93.80 & 94.80 & - \\
\hline $\mathrm{K} 3$ & 97.23 & 91.46 & 93.10 & 91.21 & - \\
\hline $\mathrm{R}$ & 10.58 & 4.38 & 2.06 & 3.59 & - \\
\hline \multicolumn{3}{|c|}{ Optimal conditions $^{a}$} & A3 & $\mathrm{C} 2$ & \\
\hline
\end{tabular}

${ }^{a} \mathrm{~A}:$ Temperature, B: Time, C: $w$ (ricinoleic acid); D: $n$ (EGBE) $: n$ (ricinoleic acid); ${ }^{b}$ Esterification yield.

由表 4 可以看出, 在离子液体 $[\mathrm{HBth}] \mathrm{HSO}_{4}$ 催化合成 蓄麻油酸乙二醇丁醚酯的实验研究中, 四种因素对蓖麻 油酸的酯化率均有影响. 反应温度是影响酯化率的最大 因素，其次为反应时间和原料物质的量比，催化剂用量 对酯化率的影响最小. 酯化反应是可逆的吸热反应，从 热力学角度分析, 升高温度能使平衡向生成物方向移 动, 因而温度是影响酯化率的主要因素. 催化剂是动力 学影响因素, 催化剂的使用可提高反应速度, 对反应平 衡没有太大影响，所以相对其它因素而言，它对酯化率 影响较小. 根据实验结果分析可知, 离子液体 [HBth]$\mathrm{HSO}_{4}$ 催化合成蓖麻油酸乙二醇丁醚酯的最佳工艺条件 为: 蓄麻油酸与乙二醇丁醚的物质的量比为 $1: 1.15$, 催化剂质量分数为蓄麻油酸的 $2 \%$, 反应时间 $5 \mathrm{~h}$, 反应 温度为 $90{ }^{\circ} \mathrm{C}$. 在此工艺条件下, 悉麻油酸乙二醇丁醚 酯的收率可达 $98.8 \%$. 


\section{6 离子液体的重复使用性能}

离子液体较传统催化剂的显著优点是可以循环使 用, 反应结束后离子液体与产物酯自动分层, 将分离出 来的离子液体减压蒸馏并充分干燥后可以多次重复使 用, 具有良好的催化效果. 在反应温度 $90{ }^{\circ} \mathrm{C}, n$ (葍麻油 酸 $): n$ (乙二醇丁醚 $)=1: 1.15$, 催化剂 $[\mathrm{HBth}] \mathrm{HSO}_{4}$ 为蓖 麻油酸重量的 $2 \%$, 反应时间 $5 \mathrm{~h}$ 的最佳反应条件下, 考 察离子液体的重复使用性能，结果如图 3.

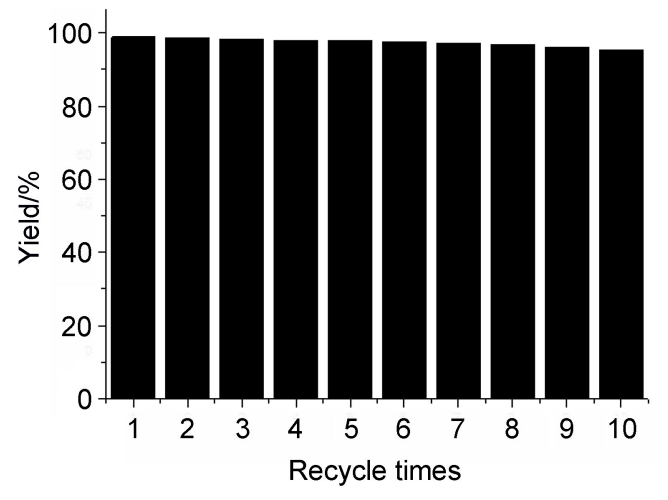

图 3 离子液体的重复使用性能

Figure 3 Reusability results of ionic liquids

从图 3 可以看出, 随着离子液体重复使用次数的增 加, 蓄麻油酸与乙二醇丁醚酯化反应的酯化率只是略有 下降. 总体上来看, 酯化反应的酯化率依然保持很高. 对重复使用 10 次的离子液体干燥处理后进行表征, 其 红外谱图中主要特征峰与最初的离子液体的红外谱图 中的特征峰基本吻合, 没有新的特征峰出现, 质谱谱图 上也没有新的杂质生成. 说明苯并噻唑离子液体不但催 化活性高, 具有良好的重复使用性能, 而且其结构还很 稳定，便于使用.

\section{7 离子液体催化合成系列蓖麻油酸二元醇梄酯和短 链脂肪酸酯}

以环已烷为带水剂, 离子液体 $[\mathrm{HBth}] \mathrm{HSO}_{4}$ 为催化 剂, 通过菎麻油酸分别与乙二醇乙醚、乙二醇丙醚、丙 二醇乙醚、丙二醇丙醚、丙二醇丁醚进行一系列酯化反 应，同时为了拓宽其应用范围，还用同样的方法合成了 系列短链脂肪酸酯, 分别是乙酸乙丙烯酸丁酯、丙烯酸 正戊酯丁二酸二乙酯、马来酸二乙酯、异戊酸丁酯, 对 酯化率进行了比较研究, 结果如表 6 .

由表 6 可知, 离子液体催化剂有较高的催化效果和 较广的应用范围, 其中合成系列蓖麻油酸二元醇醚酯反 应的酯化率均在 $95 \%$ 以上, 合成短链脂肪酸酯反应的酯 化率均比文献报道的使用其它催化剂的酯化率高 ${ }^{[32,33]}$, 特别是对含有不饱和键的脂肪酸酯化, 酯化率更高, 反 应结束后, 离子液体与相应的系列酯化产物均能自动分
表 6 离子液体催化合成系列㵧麻油酸二元醇醚 ${ }^{a}$

Table 6 Esterification results for various binary alcohol ether acids with ricinoleic acid in [HBth] $\mathrm{HSO}_{4}$

\begin{tabular}{clc}
\hline Entry & \multicolumn{1}{c}{ Product } & Yield $^{b} / \%$ \\
\hline 1 & 2-Ethoxyethanyl ricinoleate & 98 \\
2 & 2-Propoxyethanyl ricinoleate & 99 \\
3 & 1-Ethoxy-2-propanyl ricinoleate & 98 \\
4 & 1-Propoxy-2-propanyl ricinoleate & 98 \\
5 & 1-Butoxy-2-propanyl ricinoleate & 97 \\
6 & Butyl acrylate & 95 \\
7 & n-Pentyl acetate & 97 \\
8 & Diethyl succinate & 90 \\
9 & Diethyl maleate & 99 \\
10 & Butyl isovalerate & 93 \\
\hline
\end{tabular}

${ }^{a}$ Temperature $=90{ }^{\circ} \mathrm{C}, w$ (ricinoleic acid) $=2 \%, n$ (EGBE) $: n$ (ricinoleic acid) $=1.15: 1 ;{ }^{b}$ Esterification yield.

层，后处理简单，可以循环使用. 此类苯并噻唑离子液 体催化剂具有很多硫酸无法比拟的优点.

\section{2 结论}

离子液体一般包括有机阳离子和无机阴离子两大 部分，阴、阳离子可以根据需要进行配伍合成出不同酸 强度的离子液体，使用此类清洁的可再生的酸性离子液 体完全可以代替强污染性的传统工业酸催化剂, 具有突 出的节能环保优越性. 根据离子液体可设计的特点，以 苯并噻唑作为阳离子部分, 选择用不同的质子酸作为无 机阴离子部分, 合成了一系列苯并噻唑离子液体, 并用 核磁、质谱、红外对其进行了结构表征，对其酸性、熔 点、热稳定性、溶解性等理化性质进行了系统的研究, 获 得了一些规律性认识. 此类离子液体具有良好的理化性 质，可以应用到有机合成、分离纯化等多个领域. 此外, 还利用苯并噻唑离子液体对合成系列蓄麻油酸乙二醇 丁醚酯进行了应用研究. 与传统浓硫酸催化酯化相比, 该催化体系的优势在于: 首先其合成方法简单，成本也 较低，反应后通过重力沉降即可实现离子液体与产物的 自然分离，不产生废弃物; 其次具有较高的催化活性, 少量的离子液体就可以达到高酯化率，且可以多次循环 使用; 再者苯并噻唑离子液体腐蚀性比浓硫酸低, 而且 不含 $\mathrm{F} 、 \mathrm{Cl} 、 \mathrm{Br}$ 等卤素原子，不会对环境造成潜在的污 染; 还有此类离子液体在反应中不挥发, 不产生酸雾, 几乎无流失，基本实现了合成反应过程的绿色化. 综上 所述，该催化剂活性较高，成本低，使用方便，工艺简 单，废液排放少，过程清洁，对环境污染小且不腐蚀设 备, 解决了传统酸催化剂所具有的副产物多、易挥发、 腐蚀性强和用量大等缺陷，有望替代传统酸催化剂应用 于酯化反应过程, 为真正实现绿色化学工业化提供了可 靠的基础，具有十分重要的应用前景. 


\section{3 实验部分}

\section{1 仪器与试剂}

RE-2000B 型旋转蒸发器 (上海亚荣生化仪器厂); DF-101S 型集热式恒温加热磁力搅拌器(长城科工贸有 限公司); FTS-135 型傅立叶变换红外光谱仪 (美国 BIO-RAD 公司); STD-2960 型热重分析仪(德国 Netzsch 公司); PHS-25 型 $\mathrm{pH}$ 计(上海精密科学仪器有限公司); STD-2960 差热-热重联用热分析仪(美国 TA 公司); ${ }^{1} \mathrm{H}$ NMR 和 ${ }^{13} \mathrm{C}$ NMR (内标为 TMS, 溶剂为 $\mathrm{D}_{2} \mathrm{O}$ ) 使用 Bruker Ascend $400 \mathrm{MHz}$ 核磁共振仪测定; HRMS 使用 Agilent 6540 UHD Q-TOF LC/MS 型高分辨质谱仪测定.

苯并噻唑, 浓硫酸[分析纯, 大茂化学(天津)制剂有 限公司]; 高氯酸(分析纯, 天津博迪化工股份有限公司); 磷酸(分析纯, 天津市东方化工有限公司); 硝酸[分析纯, 永大化学制剂(天津)有限公司]; 乙醇(分析纯, 天津市 红岩试剂厂); 蓖麻油酸(化学纯, 国药集团化学试剂有 限公司), 其他所用的试剂均从阿拉丁试剂 公司采购.

\section{2 苯并噻唑离子液体的制备}

苯并噻坐离子液体分别参照文献[34 36], 用类似 方法合成.

在配有机械搅拌的 $250 \mathrm{~mL}$ 三口烧瓶中加入 $13.5 \mathrm{~g}$ $(0.10 \mathrm{~mol})$ 苯并噻唑和无水乙醇 $50 \mathrm{~mL}$, 将三口烧瓶置于 冰水浴中, 冷却至 $0 \sim 5{ }^{\circ} \mathrm{C}$, 在剧烈搅拌下 $30 \mathrm{~min}$ 内缓 慢滴加 $11.0 \mathrm{~g}(0.11 \mathrm{~mol})$ 的质量分数为 $98 \%$ 硫酸溶液, 然 后在室温下反应 $3 \mathrm{~h}$. 蒸除溶剂, 残余物用乙酸乙酯 $(10$ $\mathrm{mL} \times 3$ ) 洗涤, 无水乙醇重结晶, 冷却到 $0{ }^{\circ} \mathrm{C}$, 用布氏漏 斗过滤, 滤饼真空干燥一定时间后即得离子液体苯并噻 唑硫酸氢盐([HBth] $\mathrm{HSO}_{4}$, IL1), 白色针状晶体, 产率 96\%. m.p. 130.2 130.8 ${ }^{\circ} \mathrm{C} ;{ }^{1} \mathrm{H}$ NMR $\left(\mathrm{D}_{2} \mathrm{O}, 400 \mathrm{MHz}\right) \delta$ : 7.31 (t, $J=7.2 \mathrm{~Hz}, 1 \mathrm{H}), 7.37$ (t, $J=7.2 \mathrm{~Hz}, 1 \mathrm{H}), 7.62$ (d, $J=8.0 \mathrm{~Hz}, 1 \mathrm{H}), 7.74(\mathrm{~d}, J=8.0 \mathrm{~Hz}, 1 \mathrm{H}), 9.72(\mathrm{~s}, 1 \mathrm{H}) ;{ }^{13} \mathrm{C}$ NMR $\left(\mathrm{D}_{2} \mathrm{O}, 100 \mathrm{MHz}\right) \delta: 118.1,123.1,127.9,129.0$, 130.5, 141.2, 160.6; FT-IR (KBr) v: 3419, 3091, 3052, 2817, 2408, 1606, 1587, 1530, 1493, 1469, 1250, 1239, $765 \mathrm{~cm}^{-1}$; HRMS (ESI) calcd for $\mathrm{C}_{7} \mathrm{H}_{6} \mathrm{NS}\left[\mathrm{M}-\mathrm{HSO}_{4}\right]^{+}$: 136.0221, found 136.0222 .

在配有机械摚拌的 $250 \mathrm{~mL}$ 三口烧瓶中加入 $13.5 \mathrm{~g}$ (0.1 mol)苯并噻唑和无水乙醇 $50 \mathrm{~mL}$, 将三口烧瓶置于 冰水浴中, 冷却至 $5{ }^{\circ} \mathrm{C}$, 在剧烈搅拌下 $30 \mathrm{~min}$ 内滴加 $12.6 \mathrm{~g}(0.11 \mathrm{~mol})$ 的质量分数为 $85 \%$ 磷酸溶液, 然后在室 温下反应 $3 \mathrm{~h}$. 蒸除溶剂, 残余物用乙酸乙酯 $(10 \mathrm{~mL} \times 3)$ 洗涤, 无水乙醇重结晶, 冷却到 $0{ }^{\circ} \mathrm{C}$, 用布氏漏斗过 滤、而后滤饼真空干燥一定时间后即得离子液体苯并噻 唑磷酸二氢盐([HBth $\left.] \mathrm{H}_{2} \mathrm{PO}_{4}, \mathbf{I L 2}\right)$, 白色粉末状固体, 收
率 80\%. m.p. 120.2 120.9 ${ }^{\circ} \mathrm{C} ;{ }^{1} \mathrm{H}$ NMR $\left(\mathrm{D}_{2} \mathrm{O}, 400 \mathrm{MHz}\right)$ $\delta: 7.43(\mathrm{t}, J=7.2 \mathrm{~Hz}, 1 \mathrm{H}), 7.51(\mathrm{t}, J=7.2 \mathrm{~Hz}, 1 \mathrm{H}), 7.83$ (d, $J=8.0 \mathrm{~Hz}, 1 \mathrm{H}), 7.91(\mathrm{~d}, J=8.0 \mathrm{~Hz}, 1 \mathrm{H}), 9.60(\mathrm{~s}, 1 \mathrm{H}) ;{ }^{13} \mathrm{C}$ NMR $\left(\mathrm{D}_{2} \mathrm{O}, 100 \mathrm{MHz}\right) \delta$ : 119.3, 123.0, 127.4, 128.4, 131.4, 144.1, 159.7; FT-IR (KBr) v: 3446, 3136, 3069, 2970, 2882, 1748, 1645, 1563, 1543, 1491, 1489, 1041, $791 \mathrm{~cm}^{-1}$; HRMS (ESI) calcd for $\mathrm{C}_{7} \mathrm{H}_{6} \mathrm{NS} \quad\left[\mathrm{M}-\mathrm{H}_{2} \mathrm{PO}_{4}\right]$ ${ }^{+}: 136.0221$, found 136.0209 .

在配有机械搅拌的 $250 \mathrm{~mL}$ 三口烧瓶中加入 $13.5 \mathrm{~g}$ (0.1 mol)苯并噻唑和无水乙醇 $50 \mathrm{~mL}$, 将三口烧瓶置于 冰水浴中, 冷却至 $5{ }^{\circ} \mathrm{C}$, 在剧烈搅拌下 $30 \mathrm{~min}$ 内滴加 $15.7 \mathrm{~g}(0.11 \mathrm{~mol})$ 的质量分数为 $70 \%$ 高氯酸溶液, 然后在 室温下反应 $3 \mathrm{~h}$. 蒸除溶剂, 残余物用乙酸乙酯 $(10$ $\mathrm{mL} \times 3$ )洗涤, 无水乙醇重结晶, 冷却到 $0{ }^{\circ} \mathrm{C}$, 用布氏漏 斗过滤、而后滤饼真空干燥一定时间后即得离子液体苯 并噻唑高氯酸盐([HBth $\left.] \mathrm{ClO}_{4}, \mathbf{I L 3}\right)$, 白色粉末状固体, 产率 95\%. m.p. $138.2 \sim 138.8{ }^{\circ} \mathrm{C} ;{ }^{1} \mathrm{H}$ NMR $\left(\mathrm{D}_{2} \mathrm{O}, 400\right.$ MHz) $\delta$ : $7.58(\mathrm{t}, J=7.2 \mathrm{~Hz}, 1 \mathrm{H}), 7.67(\mathrm{t}, J=7.2 \mathrm{~Hz}, 1 \mathrm{H})$, $7.93(\mathrm{~d}, J=8.0 \mathrm{~Hz}, 1 \mathrm{H}), 8.04(\mathrm{~d}, J=8.0 \mathrm{~Hz}, 1 \mathrm{H}), 9.93$ (s, $1 \mathrm{H}) ;{ }^{13} \mathrm{C}$ NMR $\left(\mathrm{D}_{2} \mathrm{O}, 100 \mathrm{MHz}\right) \delta: 118.4,123.4,128.1$, 129.2, 130.8, 141.6, 160.8; FT-IR (KBr) v: 3426, 3081, 3069, 2970, 2882, 1629, 1585, 1571, 1464, 1429, 1384, $788,489 \mathrm{~cm}^{-1}$; HRMS (ESI) calcd for $\mathrm{C}_{7} \mathrm{H}_{6} \mathrm{NS}[\mathrm{M}-$ $\left.\mathrm{ClO}_{4}\right]^{+}: 136.0221$, found 136.0220 .

在配有机械搅拌的 $250 \mathrm{~mL}$ 三口烧瓶中加入 $13.5 \mathrm{~g}$ $(0.1 \mathrm{~mol})$ 苯并噻唑和无水乙醇 $50 \mathrm{~mL}$, 将三口烧瓶置于 冰水浴中, 冷却至 $5{ }^{\circ} \mathrm{C}$, 在剧烈搅拌下 $30 \mathrm{~min}$ 内滴加 $11.6 \mathrm{~g}(0.11 \mathrm{~mol})$ 的质量分数为 $60 \%$ 硝酸溶液, 然后在室 温下反应 $3 \mathrm{~h}$. 蒸除溶剂, 残余物用乙酸乙酯 $(10 \mathrm{~mL} \times 3)$ 洗涤, 无水乙醇重结晶, 冷却到 $0{ }^{\circ} \mathrm{C}$, 用布氏漏斗过 滤、而后滤饼真空干燥一定时间后即离子液体苯并噻唑 硝酸盐([HBth] $\mathrm{NO}_{3}$, IL4), 白色粉末状固体, 产率 $97 \%$. m.p. 132.3 132.5 ${ }^{\circ} \mathrm{C} ;{ }^{1} \mathrm{H}$ NMR $\left(\mathrm{D}_{2} \mathrm{O}, 400 \mathrm{MHz}\right) \delta: 7.40$ $(\mathrm{t}, J=7.2 \mathrm{~Hz}, 1 \mathrm{H}), 7.46(\mathrm{t}, J=7.2 \mathrm{~Hz}, 1 \mathrm{H}), 7.71(\mathrm{~d}, J=$ $8.0 \mathrm{~Hz}, 1 \mathrm{H}), 7.83(\mathrm{~d}, J=8.0 \mathrm{~Hz}, 1 \mathrm{H}), 9.51(\mathrm{~s}, 1 \mathrm{H}) ;{ }^{13} \mathrm{C}$ NMR $\left(\mathrm{D}_{2} \mathrm{O}, 100 \mathrm{MHz}\right) \delta$ : 118.4, 123.1, 127.8, 128.9, 130.8, 142.0, 160.4; FT-IR (KBr) v: 3432, 3136, 3074, 2970, 1676, 1573, 1568, 1491, 1976, 1384, 1144, 1118, 1087, 1036, 918, 785, $679 \mathrm{~cm}^{-1}$; HRMS (ESI) calcd for $\mathrm{C}_{7} \mathrm{H}_{6} \mathrm{NS}\left[\mathrm{M}-\mathrm{NO}_{3}\right]^{+}: 136.0221$, found 136.0221 .

辅助材料(Supporting Information) 苯并噻唑离子液 体的 ${ }^{1} \mathrm{H}$ NMR、 ${ }^{13} \mathrm{C}$ NMR、HRMS、FT-IR 的原始谱图. 这 些材料可以免费从本刊网站(http://siocjournal.cn/)上下 载. 


\section{References}

[1] Zeng, Q.-F.; Dong, G.-N. Tribol. Lett. 2013, 52, 47.

[2] Tong, L.-G.; Rong, H.; Lin, S.-J. J. Beijing Inst. Petro-Chem. Technol. 2010, 18, 58 (in Chinese). (佟拉嘎, 荣华, 林世静，北京石油化工学院学报, 2010, 18, 58.)

[3] Doudin, K.; A-Malaika, S.; Dole, H. Polym. Degrad. Stab. 2011, $96,438$.

[4] Dias, J.-M.; Araujo, J.-M.; Costa, J.-F. Energy 2013, 53, 58

[5] Amador, P.; Martinez, E.; Sanchez-Daza, O. J. Chem. Thermodyn. 2012, 50, 15 .

[6] Wu, J. Agric. Mach. 2011, 61, 61 (in Chinese). (吴杰, 农业机械, 2011, 61, 61.)

[7] Zhao, D.-B.; Fei, Z.-F.; Geldbach, T.-J.; Scopelliti, R.; Laurenczy, G.; Dyson, P.-J. J. Am. Chem. Soc. 2005, 88, 665 .

[8] Inui, K.; Kurabayashi, T.; Sato, S.; Ichikawa, N. J. Mol. Catal A: Chem. 2004, 216, 147

[9] Amador, P.; Martinez E, Sanchez-Daza O. J. Chem. Thermodyn. 2012, 50, 15.

[10] Li, Y.; Hu, S.-L.; Cheng, J.-H.; Lou, W.-Y. Chin. J. Catal. 2014, 35, 396

[11] Zhao, D.-S.; Liu, M.-S.; Ge, J.-J.; Zhang, J.; Ren, P.-B. Chin. J. Org. Chem. 2012, 32, 2382 (in Chinese). (赵地顺, 刘猛帅, 葛京京, 张娟, 任培兵, 有机化学, 2012, 32, 2382.)

[12] Hou, H. L.; Li, Z. F.; Ying, A. G. Chin. J. Org. Chem. 2014, 34, 1277 (in Chinese)

(侯海亮, 李志峰, 应安国, 许松林, 有机化学, 2014, 34, 1277.)

[13] Peter, W.; Wilhelm, K. Angew. Chem., Int. Ed. 2000, 39, 3772.

[14] Plechkova, N.-V.; Seddon, K.-R. Chem. Soc. Rev. 2008, 370, 123.

[15] Tokuda, H.; Tsuzuki, S.; Susan, M. A.-B. H.; Hayamizu, K.; Watanabe, M. J. Chin. Phys. Chem. B 2006, 110, 19593.

[16] Song, Y.-L.; Wang, X.-C.; Huang, C.-P.; Liang, F.-B.; Liu, Z.-C.; Chen, B.-H. Chin. J. Org. Chem. 2013, 33, 1715.

[17] Jankovic, M. R.; Sinadinovic-Fiser, S. V.; Govedarica, O.-M. Ind. Eng. Chem. Res. 2014, 53, 9357.

[18] Elsheikh, Y.-A. Energy Convers. Manage. 2011, 52, 804.

[19] Liu, S.-W.; Li, L.; Yu, S.-T. Chin. J. Catal. 2010, 31, 1433.

[20] Earle, M.-J.; Seddon, K.-R. Pure Appl. Chem. 2000, 72: 1391-1398.

[21] Marsh, K.-N; Boxall, J.-A.; Lichtenthaler, R. Fluid Phase Equilib. 2004, 219: 93 98 .
[22] Wu, K.; Li, C.-X. Chin. J. Org. Chem. 2011, 31, 119 (in Chinese). (吴坤, 李存雄, 有机化学, 2011, 31, 119.)

[23] Li, J.-Y.; Peng, J.-J.; Qiu, H.-Y.; Jiang, J.-X.; Wu, J.-R.; Ni, Y.; Lai, G.-Q. Chin. J. Org. Chem. 2007, 27, 483 (in Chinese). (厉嘉云, 彭家建, 邱化玉, 蒋剑雄, 邬继荣, 倪勇, 来国桥, 有 机化学, 2007, 27, 483.)

[24] Wang, Y.-Y.; Wu, Y.-T.; Liu, K.; Dai, L.-Y. Chin. Chem. Lett. 2012, 23, 1031.

[25] Venkataraman, V- R; Ali, K.-S. Indian J. Heterocycl. Chem. 2001, 11,93 .

[26] Zhao, D. S.; Liu, M. S.; Xu, Z. C.; F, J. T.; Ren, P. B. Chem. Ind. Eng. Prog. 2011, 30, 2287 (in Chinese).

(赵地顺，刘猛帅，徐智策，付江涛，任培兵，化工进展, 2011, 30, 2287.)

[27] Wasserscheid, P.; Keim, W. Angew. Chem., Int. Ed. 2000, 39, 3772.

[28] Huddleston, J. G.; Visser, A. E.; Reichert, W. M.; Willauer, H. D.; Broker, G. A.; Rogers, R. D. Green. Chem. 2001, 3, 156.

[29] Fredakle, C.-P.; Crosthwaite, J.-M.; Hert, D.-G. J. Chem. Eng. Data 2004, 49, 954

[30] Yang, L.; Lu, J.; Bai, Y. J. Chin.. J. Org. Chem. 2003, 23, 659 (in Chinese).

(杨玲, 路军, 白银娟, 有机化学, 2003, 23, 659.)

[31] Anthony, J. L.; Maginn, E. J.; Brennecke, J.-F. J. Phys. Chem. B 2001, 105, 10942.

[32] Ji, S. X.; Zhao, Y. J.; He, C. Petrochem. Technol. 2010, 39, 251 (in Chinese). (纪世雄, 赵义军, 何春, 石油化工, 2010, 39, 251.)

[33] Wang, Q.; Ma, R. X. J. Langzhou Univ. Technol. 2005, 31, 57 (in Chinese).

(王俏，马荣萱，兰州理工大学学报, 2005, 31, 57.)

[34] Zeng, H. Y.; Ying, S. F.; Li, Y. Chin. J. Org. Chem. 2007, 27, 528 (in Chinese). (曾鸿耀，尹述凡，李颖，有机化学，2007, 27, 528.)

[35] Peng, Q.; Zhang, Y. W.; Wang, X. M. Chin. J. Synth. Chem. 2012, 20, 28 (in Chinese).

(彭琦，张义文，王小敏，王卓端，姚舜，宋航，合成化学，2012， 20, 28.)

[36] Huang, B.-H.; Li, Z.-J.; Shi, N.; Xu, X.-L.; Fang, Y.-X. Chin. J. Org. Chem. 2009, 29, 770 (in Chinese).

(黄宝华, 黎子进, 史娜, 徐效陵, 张焜, 方岩雄, 有机化学, 2009, 29, 770.)

(Li, L.; Fan, Y.) 